\title{
Acúmulo de massa seca e de nutrientes em gliricídia em resposta ao estresse hídrico e a doses de fósforo ${ }^{1}$
}

\author{
Dry matter and nutrients accumulation in Gliricidia sepium in \\ response to water stress and phosphorus levels
}

\author{
Antonio Lucineudo de Oliveira Freire ${ }^{2 *}$; Douglas Alexandre Saraiva Leão; \\ José Romilson Paes de Miranda ${ }^{4}$
}

\begin{abstract}
Resumo
Dentre os fatores que mais têm limitado o crescimento vegetal e a produção agrícola nas regiões tropicais estão a baixa disponibilidade de fósforo e o estresse hídrico. Esses fatores assumem proporção ainda maior na região semiárida do Nordeste do Brasil. Em função disso, conduziu-se um experimento com o objetivo de avaliar o efeito de doses de fósforo e dois regimes hídricos nos acúmulos de massa seca da parte aérea (MSPA) e de nutrientes em plantas de gliricídia. O delineamento experimental foi o de blocos casualizados, disposto em esquema fatorial 2 x 4, correspondendo a dois regimes hídricos (com e sem estresse hídrico) e quatro doses de fósforo $\left(0,50,100\right.$ e $\left.150 \mathrm{mg} \mathrm{dm}^{-3} \mathrm{de} \mathrm{P}\right)$. As plantas cresceram em vasos plásticos contendo $9 \mathrm{~kg}$ de solo e o estresse hídrico foi imposto através da suspensão da irrigação, aos 45 dias após a emergência, permanecendo durante 15 dias nessa condição. Foram analisadas a matéria seca das plantas e os conteúdos de N, P, K, Ca, Mg, S, Fe, Mn, Cu e Zn na parte aérea. O estresse hídrico reduziu a produção de massa seca e o acúmulo de $\mathrm{N}$ e $\mathrm{Zn}$, e aumentou o acúmulo de $\mathrm{P}, \mathrm{S}$ e $\mathrm{Cu}$ na MSPA das plantas. A adubação fosfatada aumentou o acúmulo de N, P, Ca, S, Mn e Fe na MSPA das plantas.

Palavras-chave: Nutrição mineral, adubação fosfatada, leguminosa forrageira
\end{abstract}

\begin{abstract}
Among the factors that have limited plant growth and crop production in tropical regions are the low availability of phosphorus and water stress. These factures assume even greater proportion in the semiarid region of northeastern Brazil. As a result, we conducted an experiment to evaluate the effects of phosphorus and two water regimes effects in dry matter and nutrients accumulation in Gliricidia sepium plants. The experimental design was a randomized block design, arranged in a 2 x 4 factorial scheme, corresponding to two water regimes (with and without water stress) and four phosphorus levels $(0 ; 50$; 100 and $150 \mathrm{mg} \mathrm{dm}^{-3}$ of P). The plants grown in plastic pots containing $9 \mathrm{~kg}$ of soil, and the water stress was imposed by the suspension of irrigation, 45 days after emergence and remained in that condition for 15 days. It were analyzed the dry matter and $\mathrm{N}, \mathrm{P}, \mathrm{K}, \mathrm{Ca}, \mathrm{Mg}, \mathrm{S}, \mathrm{Fe}, \mathrm{Mn}, \mathrm{Cu}$ and $\mathrm{Zn}$ content in shoots. Water stress was detrimental to the production of dry matter and $\mathrm{N}$ and $\mathrm{Zn}$ accumulation, while promoted accumulation of $\mathrm{P}, \mathrm{S}$ and $\mathrm{Cu}$. The fertilization favored N, P, Ca, S, Mn, and Fe shoot accumulation.

Key words: Mineral nutrition, fertilization, forage legume
\end{abstract}

\footnotetext{
${ }^{1}$ Parte da Dissertação de Mestrado do segundo autor apresentada à Universidade Federal de Campina Grande, UFCG, como parte dos requisitos para obtenção do título de Mestre em Zootecnia.

${ }^{2}$ Eng $^{\circ}$ Agr $^{\circ}$, Prof. Dr. Associado, UFCG, Centro de Saúde e Tecnologia Rural, Av. Universitária, s/n, B. Santa Cecília, CEP 58.708110, Patos, PB. E-mail: lofreire@cstr.ufcg.edu.br

${ }^{3}$ Licenciado em Ciências Agrárias, Mestre em Zootecnia. E-mail: douglasaraiva@hotmail.com

${ }^{4}$ Eng $^{\circ}$ Agr ${ }^{\circ}$, Prof. Dr. Associado, UFCG, Centro de Desenvolvimento Sustentável do Semiárido, R. Luiz Grande, s/n, CEP 58.540000, Sumé, PB. E-mail: romilson@ufcg.edu.br

* Autor para correspondência
} 


\section{Introdução}

A gliricídia (Gliricidia sepium (Jacq.) Steud.) tem sido utilizada como cerca-viva e na produção de forragem, estacas e energia (DRUMOND; CARVALHO FILHO, 1999), além de ser empregada em sistemas agroflorestais (BARRETO; FERNANDES, 2001; QUEIROZ et al., 2007). Devido ao seu rápido crescimento, alta capacidade de regeneração e fixação biológica do nitrogênio, pode ser empregada na recuperação de áreas degradadas (ZAHAWI, 2005; PETRONE; PETRI, 2009).

A degradação ambiental é um processo que tem aumentado continuamente devido à exploração desordenada dos recursos naturais. Para a recuperação das áreas degradadas têm sido empregadas espécies vegetais, nativas ou exóticas, que possam melhorar as propriedades físicas e químicas dos solos. No entanto, para o sucesso desses programas de recuperação, é necessário o conhecimento dos fatores que podem interferir no crescimento e estabelecimento das mudas no campo (FERNANDES et al., 2000), e dentre esses estão a tolerância à seca e a exigência nutricional da espécie.

O fósforo é encontrado em concentrações muito baixas nos solos das regiões tropicais (FERNANDES et al., 2000), e em virtude da capacidade de fixação que os solos apresentam, as plantas apresentam redução no crescimento ou manifestam sintomas de deficiência do mesmo (RESENDE et al., 1999). As respostas ao fornecimento do fósforo são variáveis entre as espécies florestais nativas e exóticas (FERNANDES et al., 2000; FERNANDES et al., 2007), e são explicadas por diferenças na eficiência nutricional em relação ao nutriente. Nas regiões semiáridas, além da baixa fertilidade do solo, as plantas enfrentam períodos de deficiência hídrica constantemente, limitando o crescimento e a produtividade das plantas.

A disponibilidade hídrica é uma das principais limitações para o crescimento e a produtividade das plantas, afetando um grande número de características morfológicas e fisiológicas
(NOGUEIRA，ALBUQUERQUE; SILVA，2005; SILVA et al., 2008).

Em virtude da carência de informações sobre a resposta à adubação fosfatada aliada ao déficit hídrico, em plantas de gliricídia, conduziu-se esse trabalho no sentido de contribuir para os estudos de programas de recuperação de áreas degradadas, particularmente em regiões que apresentem déficits hídricos frequentes.

O objetivo desse trabalho foi avaliar o efeito de diferentes doses de fósforo nos acúmulos de massa seca e de macro e micronutrientes na parte aérea de plantas de gliricídia submetidas ao estresse hídrico.

\section{Material e Métodos}

O experimento foi conduzido em telado no Viveiro Florestal da Universidade Federal de Campina Grande, Campus de Patos (PB), nas coordenadas geográficas $7^{\circ} 01$ '2 $28^{\prime}$ 'S e $37^{\circ} 16^{\prime} 48^{\prime \prime} \mathrm{W}$.

Coletou-se amostra de um Neossolo litólico, na profundidade de $0-20 \mathrm{~cm}$ que, após secagem e peneiramento, foi submetida a análises químicas, revelando: $\mathrm{pH}$ em água $=5,8 ; \mathrm{H}+\mathrm{Al}=1,1 \mathrm{cmol}_{\mathrm{c}}$ $\mathrm{dm}^{-3} ; \mathrm{Ca}=6,0 \mathrm{cmol}_{\mathrm{c}} \mathrm{dm}^{-3} ; \mathrm{Mg}=1,2 \mathrm{cmol}_{\mathrm{c}} \mathrm{dm}^{-3}$; $\mathrm{K}=0,38 \mathrm{mg} \mathrm{dm}^{-3}$ e $\mathrm{P}=7,5 \mathrm{mg} \mathrm{dm}^{-3}$.

Os tratamentos foram distribuídos em delineamento inteiramente casualizado, em esquema fatorial 2 x 4, com 4 repetições, correspondendo a dois regimes hídricos (com e sem estresse hídrico) e quatro doses de fósforo $\left(0,50,100\right.$ e $150 \mathrm{mg} \mathrm{dm}^{-3}$ de P).

As plantas cresceram em recipientes plásticos contendo $9 \mathrm{~kg}$ do solo, e receberam adubação, em solução, com o $\mathrm{N}$ fornecido na forma de $\mathrm{NH}_{4} \mathrm{NO}_{3}$ (112 $\mathrm{mg} \mathrm{dm}^{-3}$ ), usando-se $36 \mathrm{~mL} /$ vaso, parcelado em três aplicações. O P, variando em função do tratamento de $\mathrm{P}$ a ser testado, foi fornecido pelo uso de $\mathrm{KH}_{2} \mathrm{PO}_{4} 1 \mathrm{M}$, sendo 14,4; 28,8 e 43,2 $\mathrm{mL} /$ vaso, respectivamente para os tratamentos 50,100 e 150 $\mathrm{mg} \mathrm{dm}{ }^{-3}$. O K foi complementado pelo uso de $\mathrm{KCl}$, variando em função do tratamento de $\mathrm{P}\left(0 \mathrm{mg} \mathrm{dm}^{-3}\right.$ 
de $\mathrm{P}=43,2 \mathrm{~mL} /$ vaso; $50 \mathrm{mg} \mathrm{dm}^{-3}$ de $\mathrm{P}=28,8 \mathrm{~mL} /$ vaso; $100 \mathrm{mg} \mathrm{dm}^{-3}$ de $\mathrm{P}=14,4 \mathrm{~mL} / \mathrm{vaso} ; 150 \mathrm{mg}$ $\mathrm{dm}^{-3}$ de $\mathrm{P}=0$ de $\mathrm{KCl}$ ).

No ato da semeadura foram colocadas cinco sementes por vaso, sendo realizado um desbaste aos 15 dias após a emergência (DAE), deixandose duas plantas por vaso. A irrigação dos vasos foi efetuada diariamente, com base no peso dos vasos, procurando manter o teor de umidade do solo próximo de $60 \%$ da capacidade de campo. Decorridos 45 DAE, as plantas foram separadas em dois grupos, com 16 vasos cada, sendo que um grupo recebeu irrigação normalmente (tratamento sem estresse hídrico), enquanto que outro teve a irrigação suspensa (tratamento com estresse hídrico). O estresse hídrico foi imposto até que fosse observada a parada do crescimento das plantas e o enrolamento das folhas do ápice, que aconteceu 15 dias após a suspensão da irrigação.

Ao final do experimento (60 DAE) as plantas foram colhidas e separadas em parte aérea e raízes. O material foi colocado para secar em estufa de circulação forçada de ar a $\pm 65^{\circ} \mathrm{C}$, até atingirem massa constante, para obtenção da massa seca da parte aérea e das raízes. Após secagem, a parte aérea foi moída para a realização das análises químicas. No extrato obtido após a digestão nitroperclórica, foram determinados os teores de $\mathrm{P}$ por colorimetria, de K por fotometria de chama, de S por turbidimetria, e de $\mathrm{Ca}, \mathrm{Mg}, \mathrm{Fe}, \mathrm{Zn}$ e $\mathrm{Mn}$ por espectrofotometria de absorção atômica, seguindo-se metodologia descrita por Malavolta, Vitti e Oliveira (1997). Os teores de $\mathrm{N}$ foram determinados pelo método de Kjeldahl (LIAO, 1981), sendo a destilação e a titulação realizadas segundo Bremner e Edwards (1965).

Os dados foram submetidos à análise de variância e ajustados por equações de regressão.

\section{Resultados e Discussão}

Não houve interação significativa entre doses de $\mathrm{P}$ e regimes hídricos em nenhum dos parâmetros avaliados. Quanto aos regimes hídricos, verificouse efeito significativo na massa seca da parte aérea e nos conteúdos de N, P, S, Cu e Zn. Em relação às doses de $\mathrm{P}$, houve efeito significativo na massa seca das raízes, na razão parte aérea/raiz, e nos conteúdos de N, P, Ca, S, Fe, Mn e Zn.

A massa seca da parte aérea (MSPA) das plantas foi reduzida com o estresse hídrico (Tabela 1), provavelmente devido à baixa produção de fotoassimilados causada pela redução na disponibilidade de água para as plantas (MATSUI; SINGH, 2003). O estresse hídrico causa reduções no potencial hídrico foliar, transpiração (KUMAR; SHARMA, 2009), e na atividade das enzimas do Ciclo de Calvin (MONAKHOVA; CHERNYADÉV, 2004), reduz a taxa fotossintética (FU; HUANG, 2001), além de alterar a partição de assimilados entre as partes das plantas (KUMAR; SHARMA, 2009), resultando em menor produção de massa seca. Kumar e Sharma (2009) observaram que plantas de Vigna radiata cultivadas no campo e submetidas ao estresse hídrico através da suspensão da irrigação acumularam maior quantidade de massa seca na parte aérea em relação às raízes quando submetidas ao estresse hídrico. Além disso, observaram que o estresse hídrico reduziu a massa seca das folhas, hastes, vagens e grãos. Em plantas de amendoim, gergelim e mamona, cultivadas em vaso e submetidas a diferentes ciclos de deficiência hídrica, Pinto et al. (2008) observaram limitações no acúmulo de massa seca na parte aérea, causadas pela deficiência hídrica.

Em relação ao acúmulo de nutrientes na parte aérea das plantas (Tabela 1), percebeuse que o estresse hídrico causou redução no $\mathrm{N}$ e, contrariamente, aumentou o conteúdo de $\mathrm{P}$, S, Cu e Zn. Segundo Munson e Nelson (1973), quando as plantas estão sob estresse hídrico, elementos como $\mathrm{P}$ e $\mathrm{K}$, que se translocam para a raiz por difusão, podem tornar-se limitados, mesmo estando o solo bem suprido dos mesmos. Consequentemente, a deficiência hídrica do solo leva obrigatoriamente à deficiência de nutrientes, 
fato, porém, não observado nesse trabalho, o que pode ser explicado pelo efeito diluição, em que a concentração de nutrientes é diluída em virtude do maior crescimento da planta (JARREL; BEVERLY, 1981). Ao contrário do que foi obtido nesse trabalho, Silva et al. (2000) verificaram redução no acúmulo de macro e micronutrientes na parte aérea de duas espécies de eucaliptos submetidas ao déficit hídrico apresentaram.

Quanto aos tratamentos com P, houve redução linear na massa seca das raízes (MSR) e aumento também linear na razão parte aérea/raiz (RPA/R) à medida que se aumentou a doses de $\mathrm{P}$ (Figura 1). No entanto, a redução na MSR foi pequena
(23\%), quando se compara a testemunha com a dose de $150 \mathrm{mg} \mathrm{dm}^{-3}$ de P. Discordando desses resultados, Santos et al. (2008) verificaram aumento na massa seca das raízes de espécies florestais com a elevação das doses de P.O aumento na $\mathrm{RPA} / \mathrm{R}$ evidencia aumento na massa seca da parte aérea em detrimento das raízes e, nesse contexto, Resende et al. (1999) afirmaram que a partição de fotoassimilados entre raízes e parte aérea, tem sido utilizada como indicadora do status nutricional das plantas em relação ao suprimento de $\mathrm{P}$, onde, em geral, à medida que se aumenta a disponibilidade do nutriente no substrato, o crescimento da parte aérea é favorecido em detrimento do sistema radicular.

Tabela 1. Massa seca da parte aérea (MSPA) e conteúdo de nutrientes na parte aérea de plantas de gliricídia, com e sem estresse hídrico.

\begin{tabular}{lrrrrrr}
\hline Regime hídrico & $\begin{array}{c}\text { MSPA } \\
\text { g planta }^{-1}\end{array}$ & $\mathrm{~N}$ & $\begin{array}{c}\mathrm{P} \\
\mathrm{mg} \mathrm{planta}^{-1}\end{array}$ & $\mathrm{~S}$ & $\mathrm{Cu}$ & $\mathrm{Zn}$ \\
\cline { 3 - 7 } & $9,53 \mathrm{a}$ & $0,286 \mathrm{a}$ & $0,030 \mathrm{~b}$ & $0,014 \mathrm{~b}$ & $0,067 \mathrm{~b}$ & $0,084 \mathrm{~b}$ \\
Sem estresse hídrico & $7,11 \mathrm{~b}$ & $0,254 \mathrm{~b}$ & $0,038 \mathrm{a}$ & $0,019 \mathrm{a}$ & $0,094 \mathrm{a}$ & $0,129 \mathrm{a}$ \\
\hline
\end{tabular}

Figura 1. Massa seca das raízes (a) e razão parte aérea/raiz (b) de plantas de gliricídia em função das doses de P.
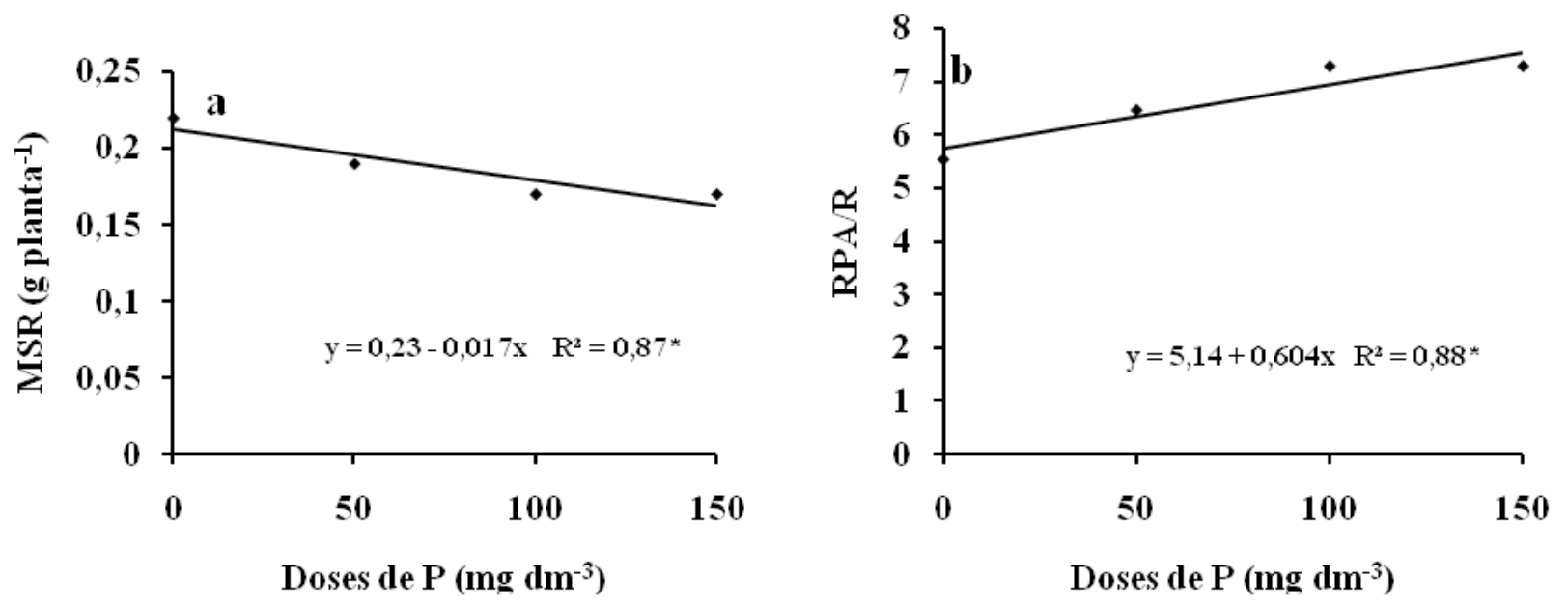

Fonte: LEÃO, 2006. 
Com aumento nas doses de $\mathrm{P}$, verificou-se comportamento linear no conteúdo dos nutrientes na parte aérea das plantas, com aumento em $\mathrm{N}, \mathrm{P}$, Ca, S (Figura 2), Fe e Mn (Figura 3), e redução em Zn. Pelos resultados verifica-se que a gliricídia responde ao fornecimento de fósforo, com aumento nas absorções de Mn (143\%), Ca (104\%), N (82\%), S $(24 \%), P(16 \%)$ e Fe (12\%), e redução no $\mathrm{Zn}$ $(12 \%)$, quando se compara o tratamento controle com a maior dose de P. As respostas de espécies florestais à adubação fosfatada são variadas, e essa variação é atribuída ao estágio sucessional e ao padrão de crescimento, em que as espécies de clímax de crescimento lento respondem muito pouco à adubação (MARSCHNER, 1991) e as espécies pioneiras respondem melhor ao fornecimento de nutrientes (RESENDE et al., 1999). Concordando com os resultados obtidos neste estudo, Fernandes et al. (2007) verificaram aumentos nos acúmulos de $\mathrm{P}, \mathrm{Ca}$ e $\mathrm{S}$ em plantas de Cordia goeldiana (freijó) à medida em que aumentou-se o fornecimento de P. Trabalhando com adubação em aveia-preta (Avena strigosa), Nakagawa, Crusciol e Zucareli (2009) observaram que os teores de $\mathrm{Ca}, \mathrm{Mg}, \mathrm{S}, \mathrm{Fe}, \mathrm{Mn}$, $\mathrm{Cu}$ e $\mathrm{Zn}$ na folha bandeira dessas plantas foram favorecidos pela adubação fosfatada. Aumentos do acúmulo de nutrientes em função de doses de $\mathrm{P}$ também foram observados por Fernandes et al. (2000) e Resende et al. (2000). Diferentemente do observado, Prado, Vale e Romualdo (2005), trabalhando com maracujazeiro amarelo, observaram que o aumento das doses de fósforo no substrato promoveu efeito linear decrescente no acúmulo de enxofre na parte aérea da planta.

Figura 2. Conteúdo de N (a), P (b), Ca (c) e S (d) na parte aérea de plantas de gliricídia em função das doses de P.
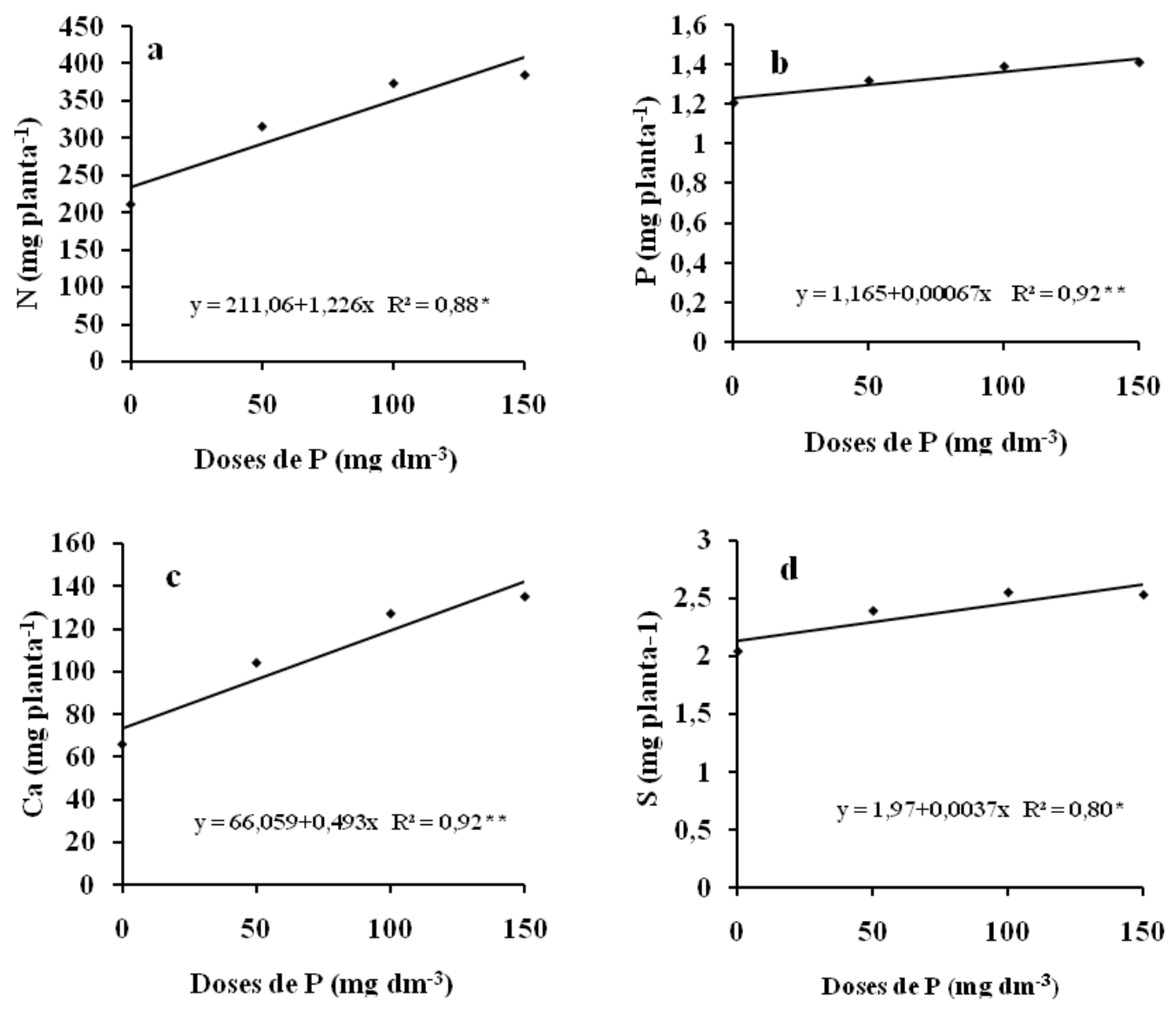

Fonte: LEÃO, 2006. 
Figura 3. Conteúdo de Fe (a), Mn (b) e Zn (c) na parte aérea de plantas de gliricídia em função das doses de P.
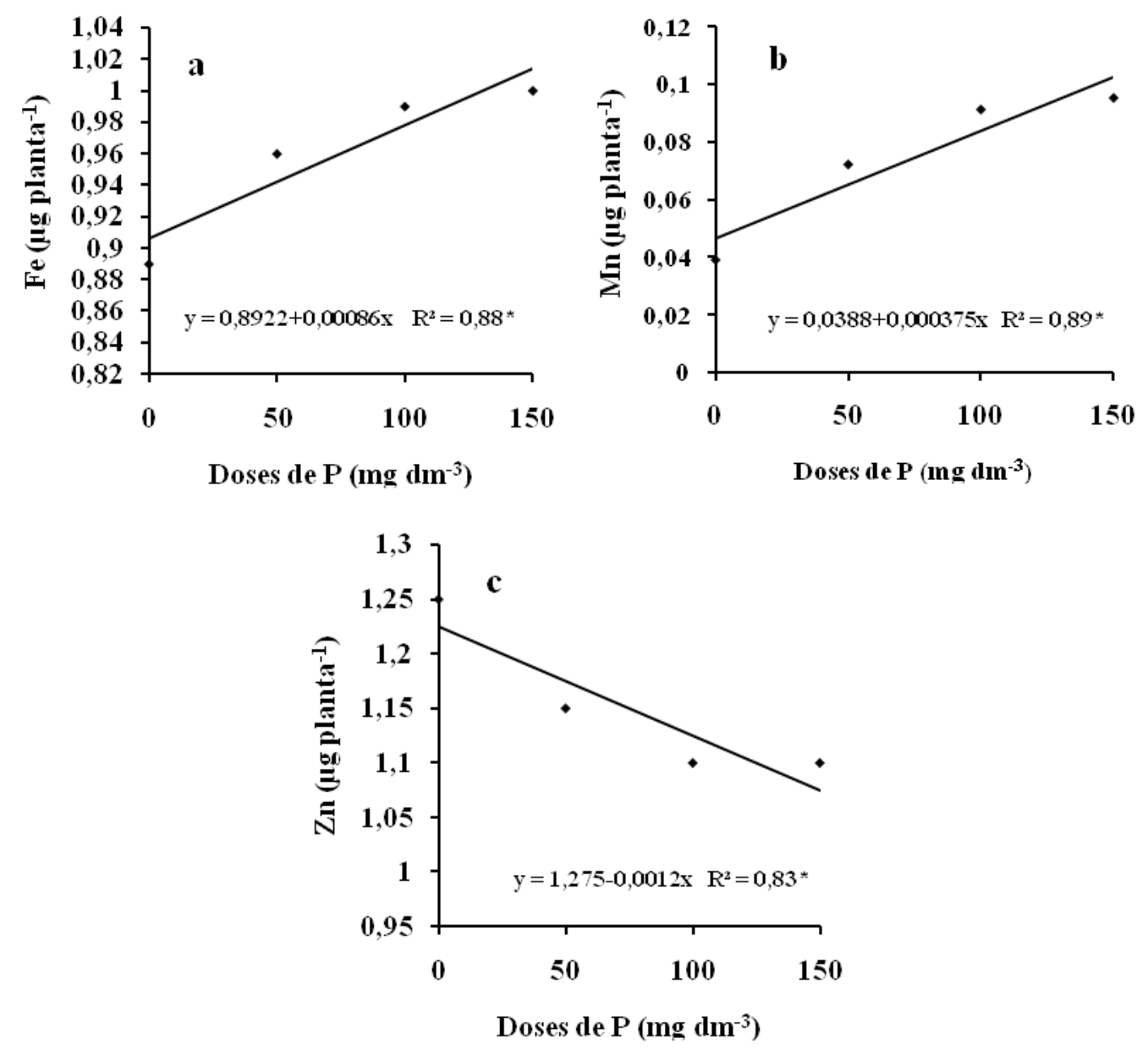

Fonte: LEÃO, 2006.

A redução no acúmulo de Zn (Figura 3c) pode ser devido à sua insolubilização causada pelo fosfato na superfície das raízes (MALAVOLTA, VITTI; OLIVEIRA, 1997; MENGEL; KIRKBY, 2001), reduzindo sua absorção, ou por uma inibição não competitiva da absorção do $\mathrm{Zn}$ pelo $\mathrm{P}$, conforme afirmam Malavolta, Vitti e Oliveira (1997). Em plantas de milho cultivadas num Argissolo Vermelho típico, na região do Cerrado, Carneiro verificaram redução nos teores foliares de $\mathrm{Zn}$ nos tratamentos que receberam superfosfato triplo. Em condições de campo, Coutinho et al. (1987) verificaram que a aplicação de doses elevadas de $\mathrm{P}$ reduziu as concentrações de $\mathrm{Zn}$ nas folhas de sorgo, sendo esse resultado atribuído ao "efeito de diluição". Contrariamente, Fernandes et al. (2007) observaram aumento no acúmulo de $\mathrm{Z}$ em função da adubação fosfatada, e atribuíram esse resultado ao maior crescimento das plantas. Na cultura do milho, Coutinho et al. (1991), empregando doses até $200 \mathrm{~kg}$ $\mathrm{ha}^{-1}$ de $\mathrm{P}_{2} \mathrm{O}_{5}$, não observaram alterações significativas nos teores de $\mathrm{Zn}$ nas folhas, se comparadas às doses mais elevadas de $\mathrm{P}$ e a testemunha.

Muitas pesquisas têm referenciado que a interação do fósforo com o zinco pode afetar a absorção, a translocação e a concentração desses nutrientes nos tecidos vegetais, provocando relações inadequadas entre os mesmos. Olsen (1972) propõe 
que o desequilíbrio entre o $\mathrm{P}$ e o $\mathrm{Zn}$, em função de respectivas concentrações excessivas, interfere na função metabólica do zinco ou do $\mathrm{P}$, em certos sítios celulares, podendo causar diminuição da taxa de translocação dos mesmos. Segundo a absorção de zinco pode diminuir devido à sua precipitação com fosfato de zinco.

\section{Conclusões}

O estresse hídrico foi prejudicial à produção de massa seca e ao acúmulo de $\mathrm{N}$ e Zn na parte aérea, enquanto estimulou o acúmulo de $\mathrm{P}, \mathrm{S}$ e $\mathrm{Cu}$.

A adubação fosfatada favoreceu o acúmulo de N, $\mathrm{P}, \mathrm{Ca}, \mathrm{S}, \mathrm{Mn}$, e Fe na parte aérea das plantas.

\section{Agradecimentos}

À Coordenação de Aperfeiçoamento de Pessoal de Ensino Superior - CAPES, pela concessão da bolsa de estudos.

\section{Referências}

BARRETO, A. C.; FERNANDES, M. F. Cultivo de Gliricidia sepium e Leucaena leucocephala em alamedas visando a melhoria dos solos dos tabuleiros costeiros. Pesquisa Agropecuária Brasileira, Brasília, v. 36, n. 10, p. 1287-1293, 2001.

BREMNER, J. M.; EDWARDS, A. P. Determination and isotope ratio analysis of different forms of nitrogen in soils. Apparatus and procedures for distillation and determination for ammonium. Soil Science Society of American Proceedings, Madison, v. 29, n. 5, p. 504-507, 1965.

COUTINHO, E. L. M.; NATALE, W.; STUPIELLO, J. J.; CARNIER, P. E. Avaliação da eficiência agronômica de fertilizantes fosfatados para a cultura do milho. Cientifica, Fortaleza, v. 19, n. 2, p. 93-104, 1991.

COUTINHO, E. L. M.; NEPTUNE, A. M. L.; SOUZA, E. C. A.; GIMENES, J. D.; NATALE, W.; BANZATTO, D. A. Diagnose da nutrição fosfatada na cultura do sorgo sacarino. Pesquisa Agropecuária Brasileira, Brasília, v. 22, n. 3, p. 451-457, 1987.
DRUMOND, M. A.; CARVALHO FILHO, O. M. de. Introdução e avaliação de Gliricidia sepium na região semi-árida do Nordeste Brasileiro. In: QUEIRÓZ, M. A. de; GOEDERT, C. O.; RAMOS, S. R. R. (Ed.). Recursos genéticos e melhoramento de plantas para o Nordeste brasileiro Versão 1.0. Petrolina, PE: Embrapa SemiÁrido /Brasília, DF. 1999. (on line).

FERNANDES, A. R.; PAIVA, H. N.; CARVALHO, J. G.; MIRANDA, J. R. P. Crescimento e absorção de nutrientes por mudas de freijó (Cordia goeldiana Huber) em função de doses de fósforo e de zinco. Revista Árvore, Viçosa, v. 31, n. 4, p. 599-608, 2007.

FERNANDES, L. A.; FURTINI NETO, A. E.; FONSECA, F. C.; VALE, F. R. Crescimento inicial, níveis críticos de fósforo e frações fosfatadas em espécies florestais. Pesquisa Agropecuária Brasileira, Brasília, v. 35, n. 6, p. 1191-1198, 2000.

FU, J.; HUANG, B. Involvement of antioxidants and lipid peroxidation in the adaptation of two cool-season grasses to localized drought stress. Environmental and Experimental Botany, Amsterdan, v. 45, n. 2, p. 105-114, 2001.

JARREL, W. M.; BEVERLY, R. B. The dilution effect in plant nutrition studies. Advances in Agronomy, Madison, v. 34, p. 197-224, 1981.

KUMAR, A.; SHARMA, K. D. Physiological responses and dry matter partitioning of summer mungbean (Vigna radiata L.) genotypes subjected to drought conditions. Journal of Agronomy and Crop Science, Berlin, v. 195, n. 4, p. 270-277, 2009.

LEÃO, D. A. S. Estresse hídrico e adubação fosfatada no desenvolvimento inicial e na qualidade da forragem da gliricídia (Gliricidia sepium (Jacq.) Steud.) e do sorgo (Sorghum bicolor (L.) Moench.). 2006. Dissertação (Mestrado em Zootecnia) - Universidade Federal de Campina Grande, Patos.

LIAO, C. F. H. Devards alloy method for total nitrogen determination. Soil Science Society of American Journal, Madison, v. 45, n. 5, p. 852-855, 1981.

MALAVOlTA, E.; VITTI, E. C.; OLIVEIRA, S. A. Avaliação do estado nutricional das plantas: princípios e aplicações. 2. ed. Piracicaba: Associação Brasileira para Pesquisa da Potassa e do Fosfato, 1997. 319 p.

MARSCHNER, H. Mechanisms of adaptation of plants to acid soils. Plant and Soil, Dordrecht, v. 134, n. 1, p. 1-20, 1991.

MATSUI, T.; SINGH, B. B. Roots characteristics in cowpea related to drought tolerance at the seedling stage. Experimental Agriculture, Cambridge, v. 39, n. 1, p. 2938, 2003. 
MENGEL, K.; KIRKBY, E. A. Principles of plant nutrition. Dordrecht: Kluwel, Academic Publishers, $2001.687 \mathrm{p}$.

MONAKHOVA, O. F.; CHERNYADÉV, II. Effects of cytokinin preparations on the stability of the photosynthetic apparatus of two wheat cultivars experiencing water deficiency. Applied of Biochemistry and Microbiology, Moscou, v. 40, n. 6, p. 659-667, 2004.

MUNSON, R. D.; NELSON, W. L. Principles and practices in plant analysis. In: WALSH, L. M.; BEATON, I. D. Soil testing and plant analysis. Madison: Soil Science Society of American Journal, 1973. p. 223-248.

NAKAGAWA, J.; CRUSCIOL, C. A. C.; ZUCARELI, C. Teores de nutrientes da folha bandeira e grãos de aveia-preta em função da adubação fosfatada e potássica. Semina: Ciências agrárias, Londrina, v. 30, n. 4, p. 833840, 2009.

NOGUEIRA, R. J. M. C.; ALBUQUERQUE, M. B.; SILVA, E. C. Aspectos ecofisiológicos da tolerância à seca em plantas da caatinga. In: NOGUEIRA, R. J. M. C.; ARAÚJO, E. L.; WILLADINO, L. G.; CAVALCANTE, U. M. T. Estresses ambientais: danos e benefícios em plantas. Recife: UFRPE, Imprensa Universitária, 2005. p. 22-31.

OLSEN, S. R. Micronutrient interactions. In: MORTVEDT, J. J.; GIORDANO, P. M.; LINDSAY, W. L. (Ed.). Micronutrients in agriculture. Madison: Soil Science Society of America, 1972. p. 243-264.

PETRONE, A.; PETRI, F. Soil bio-engineering for risk mitigation and environmental restoration in a humid tropical area. Hidrology and Earth System Sciences Discussions, Lancaster, v. 6, p. 5139-5176, 2009.

PINTO, C. M.; TÁVORA, F. J. F. A.; BEZERRA, M. A.; CORREAA, M. C. M. Crescimento, distribuição do sistema radicular em amendoim, gergelim e mamona a ciclos de deficiência hídrica. Ciência Agronômica, Fortaleza, v. 39, n. 3, p. 429-436, 2008.
PRADO, R. M.; VALE, D. W.; ROMUALDO, L. M. Fósforo na nutrição e produção de mudas de maracujazeiro. Acta Scientiarum, Agronomia, Maringá, v. 27, n. 3, p. 493-498, 2005.

QUEIROZ, L. R.; COELhO, F. C.; BARROSO, D. G.; QUEIROZ, V. A. V. Avaliação da produtividade de fitomassa e acúmulo de N, $\mathrm{P}$ e $\mathrm{K}$ em leguminosas arbóreas no sistema de aléias, em Campos dos Goytacazes, RJ. Revista Árvore, Viçosa, v. 31, n. 3, p. 383-390, 2007.

RESENDE, A. V.; FURTINI NETO, A. E.; CURI, N.; MUNIZ, J. A.; FARIA, M. R. Acúmulo e eficiencia nutricional de macronutrientes por espécies florestais de diferentes grupos sucessionais em resposta à fertilização fosfatada. Ciência e Agrotecnologia, Lavras, v. 24, n. 1, p. 160-173, 2000.

RESENDE, A. V.; FURTINI NETO, A. E.; MUNIZ, J. A.; CURI, N.; FAQUIN, V. Crescimento inicial de espécies florestais de diferentes grupos sucessionais em resposta a doses de fósforo. Pesquisa Agropecuária Brasileira, Brasília, v. 34, n. 11, p. 2071-2081, 1999.

SANTOS, J. Z. L.; RESENDE, A. V.; FURTINI NETO, A. E.; CORTE, E. F. Crescimento, acúmulo de fósforo e frações fosfatadas em mudas de sete espécies arbóreas nativas. Revista Árvore, Viçosa, v. 32, n. 5, p. 799-807, 2008.

SILVA, M. A.; SILVA, J. A. G.; ENCISO, J.; SHARMA, V.; JIFON, J. Yield components as indicators of drought tolerance of sugarcane. Scientia Agricola, Piracicaba, v. 65, n. 6, p. 620-627, 2008.

SILVA, W.; SILVA, A. A.; SEDIYAMA, T.; FREITAS, R. S. Absorção de nutrientes por mudas de duas espécies de eucalipto em resposta a diferentes teores de água no solo e competição com plantas de Brachiaria brizantha. Ciência e Agrotecnologia, Lavras, v. 24, n. 1, p. 147-159, 2000.

ZAHAWI, R. A. Establishment and growth of living fence species: an overlooked tool for the restoration of degraded areas in the tropics. Restoration Ecology, Washington, v. 13, n. 1, p. 92-102, 2005. 